\title{
LifeStream: A High-Performance Stream Processing Engine for Periodic Streams
}

\author{
Anand Jayarajan \\ University of Toronto, Vector Institute \\ Canada \\ anandj@cs.toronto.edu \\ Andrew Goodwin \\ SickKids Hospital, University of Sydney \\ Canada, Australia \\ andrew.goodwin@sickkids.ca
}

\author{
Kimberly Hau \\ University of Toronto \\ Canada \\ kimberly.hau@mail.utoronto.ca \\ Gennady Pekhimenko \\ University of Toronto, Vector Institute \\ Canada \\ pekhimenko@cs.toronto.edu
}

\begin{abstract}
Hospitals around the world collect massive amounts of physiological data from their patients every day. Recently, there has been an increase in research interest to subject this data to statistical analysis to gain more insights and provide improved medical diagnoses. Such analyses require complex computations on large volumes of data, demanding efficient data processing systems. This paper shows that currently available data processing solutions either fail to meet the performance requirements or lack simple and flexible programming interfaces. To address this problem, we propose LifeStream, a high-performance stream processing engine for physiological data. LifeStream hits the sweet spot between ease of programming by providing a rich temporal query language support and performance by employing optimizations that exploit the periodic nature of physiological data. We demonstrate that LifeStream achieves end-to-end performance up to $7.5 \times$ higher than state-ofthe-art streaming engines and $3.2 \times$ than hand-optimized numerical libraries on real-world datasets and workloads.
\end{abstract}

\section{CCS CONCEPTS}

- Information systems $\rightarrow$ Data streaming; Stream management; Data stream mining; $D B M S$ engine architectures.

\section{KEYWORDS}

stream data analytics, temporal query processing, physiological data, locality tracing, event lineage tracking, targeted query processing

\section{ACM Reference Format:}

Anand Jayarajan, Kimberly Hau, Andrew Goodwin, and Gennady Pekhimenko. 2021. LifeStream: A High-Performance Stream Processing Engine for Periodic Streams. In Proceedings of the 26th ACM International Conference on Architectural Support for Programming Languages and Operating Systems (ASPLOS '21), April 19-23, 2021, Virtual, USA. ACM, New York, NY, USA, 16 pages. https://doi.org/10.1145/3445814.3446725

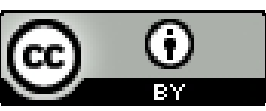

This work is licensed under a Creative Commons Attribution International 4.0 License. ASPLOS '21, April 19-23, 2021, Virtual, USA

(C) 2021 Copyright held by the owner/author(s).

ACM ISBN 978-1-4503-8317-2/21/04.

https://doi.org/10.1145/3445814.3446725

\section{INTRODUCTION}

In recent years, the healthcare industry has been experiencing an increasing trend in the adoption of approaches like data-driven diagnostic methods [13, 47], automated patient monitoring systems [22], and AI-assisted risk prediction models [2, 20, 52]. Advancements in the data collection technologies [15] and recent developments in fields like statistics and machine learning $[32,55]$ are the major enabling factors for this shift from the traditional methods used in healthcare practices. Hospitals collect and store hundreds of gigabytes of physiological data every day with the help of monitoring devices (e.g., Philips IntelliVue [44]) attached to the patients in the intensive care units (ICUs) [7, 16]. The monitors continuously collect physiological signals or waveforms such as arterial blood pressure (ABP), electrocardiogram (ECG), and electroencephalogram (EEG) and periodically produce output at regular intervals in a streaming manner. A single measurement or event in the waveform data typically contains a timestamp and a measurement value at that moment in time.

Traditionally, this data has been monitored and analyzed manually by the clinicians. However, statistical and machine learningbased algorithms can provide insights into complex data patterns that can help clinicians prepare a more precise diagnosis and personalized treatment plan [26]. Moreover, statistical models are shown to accurately predict short and long-term trends in the physiological data such as cardiac arrest [52] and sepsis risk [12]. Even though data analytics on physiological data show great potential, several practical challenges need to be addressed to unleash its full potential.

Unlike other streaming datasets, raw physiological data has a high degree of noise and discontinuities. Therefore, the data needs to go through a series of data cleaning operations and transformations (e.g., normalization and frequency filtering) before it can be used for meaningful analyses. Additionally, in certain cases, researchers need to compute derived variables from the raw data (e.g, measuring heart rate from ECG signal or finding temporal correlation between multiple signals). Although general-purpose stream processing engines that can handle these types of computations do exist in both industry and academia (e.g., Apache Spark streaming [59], Apache Beam [3], and Apache Flink [10]), we observe that they fail to be a good fit for processing physiological data for the following reason. 
Most contemporary streaming engines provide simple and flexible programming interfaces with an implicit notion of event time and support for fine-grained windowing strategies that are well suited for building physiological data processing pipelines. However, most of them are designed with a distributed setup in mind and, unfortunately, exhibit poor single machine performance [27]. This is generally compensated by scaling up the computation to large machine clusters that most hospitals neither have the infrastructure nor the required expertise to operate. Using third-party cloud services is also not widely preferred because of concerns surrounding patient privacy protection [19], security breaches [9, 53], and unpredictable downtime [45]. This necessitates on-premise computations over limited hardware resources. Our experiments reveal that most of the contemporary streaming engines fail to provide good performance under such hardware resource-constrained setup (see Section 3 for more details).

Because of these limitations, data scientists usually prefer to write adhoc data processing pipelines using numerical libraries (e.g., NumPy [37], SciPy [38] and Scikit-learn [42]). Even though, numerical libraries provide a rich set of operations for scientific computing with efficient hand-tuned implementations, the lack of temporal ordering and windowing support, as well as the absence of the unified API specifications and common data abstraction limits data scientists' ability to efficiently program and maintain large data processing pipelines. Additionally, as the pipeline gets longer and more complex, the combined workflow's performance deteriorates due to expensive data movement across the functions and lack of cross-operation optimizations [40].

Our goal in this work is to build a physiological data processing system that is both easy to program and provides high performance even under hardware resource constraints. To this end, we propose LifeStream, a new high performance stream processing engine for physiological waveform data with a rich temporal query language support. LifeStream provides high performance with efficient hardware utilization using optimizations that exploit the periodic nature of the physiological waveform data. We derive the following two key properties of temporal operations on a periodic stream:

Linearity property: The timestamp of the events produced by a temporal operator is a linear transformation of that of the input events. This property lets LifeStream map the events in an operator's output stream to its parent events in the input stream(s). Since all the temporal operations follow this property, the mapping can be extended to the entire lineage of every event produced during the query execution. We call this mechanism event lineage tracking.

Bounded memory footprint: The memory footprint of a temporal operator is bounded by the size of its input(s). Every temporal operator operates on a fixed interval size over its input streams at any given time. Since the streams' events are generated at a constant frequency, the total memory required to execute that operation can be calculated statically.

We use the above properties and propose the following three query compile-time and runtime optimizations:

(1) Locality tracing: LifeStream prepares a query execution plan that maximizes the end-to-end cache locality of the pipeline by performing static analysis on the query using periodic streams' linearity property.
(2) Static memory allocation: LifeStream estimates the upper bound of the memory required for each operation in the pipeline using the bounded memory footprint property and pre-allocates the memory for all intermediate results produced in the stream, thus almost eliminating the runtime memory allocation and deallocation overhead.

(3) Targeted query processing: We observe that the discontinuities in the physiological data are highly uneven across different signals and the number of mutually overlapping events are generally far fewer than the total number of events in the streams. Therefore, joining multiple streams together filters out many events, rendering any prior computation on them wasteful. LifeStream eliminates redundant computations using event lineage tracking mechanisms at runtime by selectively targeting only regions of input data expected to produce an output.

We evaluate LifeStream against state-of-the-art streaming engines and numerical libraries on real data analytic use cases and datasets used at The Hospital for Sick Children (SickKids) ${ }^{1}$, Canada. On a single machine, LifeStream exhibits up to $7.5 \times$ higher end-toend performance compared to the state-of-the-art streaming engine called Trill [11], and $3.2 \times$ compared to the hand-tuned numerical libraries such as SciPy [38], NumpPy [37], and Scikit-learn [42]. At the same time, LifeStream supports a rich temporal query language that is suitable for writing diverse sets of physiological data processing pipelines based on a qualitative assessment conducted on three real-world use cases. LifeStream extends the traditional query language vocabulary supported in contemporary stream processing engines to cater to certain domain-specific use cases found in the physiological data processing domain (e.g., artifact/shape detection in the signal stream). Finally, we note that even though LifeStream is built for stream processing on physiological data, the ideas proposed in this paper can also be applied to other streaming use cases where data is produced at fixed intervals.

In summary, this paper makes the following contributions:

- We showcase the challenges faced in the domain of physiological data processing and propose solutions that are evaluated on real datasets and workloads used in major hospitals.

- We derive two key properties of temporal operations on periodic streams, namely linearity and bounded memory footprint, and leverage them to propose three key optimizations, namely locality tracing, static memory allocation, and targeted query processing, that can significantly improve the hardware utilization and query execution performance compared to the state-of-the-art streaming engines.

- We propose LifeStream, a new high-performance stream processing engine with rich temporal query language support. We show that LifeStream can outperform state-of-the-art streaming engines by as much as $7.5 \times$ and hand-optimized numerical libraries by as much as $3.2 \times$ on the end-to-end data processing pipeline on real physiological datasets.

${ }^{1}$ Canada's largest pediatric hospital and the world's largest pediatric health research centre. 


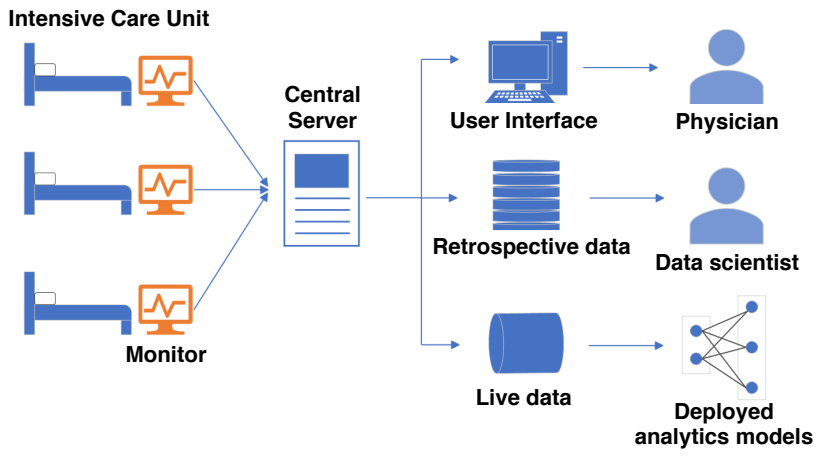

Figure 1: Typical physiological data collection infrastructure in hospitals

\section{PHYSIOLOGICAL DATA COLLECTION AND PROCESSING}

Figure 1 shows a typical physiological data collection process from the patients in intensive care units (ICUs) to keep track of their health status with the help of multiple monitoring devices [44] attached to patients, each making different measurements such as arterial blood pressure (ABP), electrocardiogram (ECG), and electroencephalogram (EEG). These devices generate continuous streams of signal events at constant intervals, typically at a rate ranging from $10^{-4} \mathrm{~Hz}$ to $10^{3} \mathrm{~Hz}$. Each signal event constitutes a timestamp corresponding to the time of measurement and a signal value, which is the magnitude of the measurement at that point in time. We use the term period to refer to the shortest time interval between consecutive events in a signal. For example, a $500 \mathrm{~Hz}$ signal stream would have a period of $2 \mathrm{~ms}$.

Unlike other streaming datasets, physiological waveform data is known to contain a high degree of noise and many discontinuities because of external disruptions in the connection between the monitoring devices and the patients. Figure 2 shows the discontinuities in the ECG and ABP signals collected from a single monitoring device over 6 months. Such disruptions are common, making it virtually impossible to run meaningful analyses on top of raw data. Therefore, the data has to go through a series of transformations and data cleaning operations before a data scientist can run any data analysis. For example, standard signal processing operations like frequency-based filtering [46] are used for removing the noise from the signals, signal value imputation methods [57] are used to fill small discontinuities in the data stream with dummy values, and data normalization methods are used to convert all signals to a uniform scale. Additionally, different data analytics algorithms might require additional variables derived from the raw data (e.g., the systolic and diastolic blood pressure [8], heart rate measured from ECG, and the temporal correlation of different signals).

Figure 3 shows a sample data processing pipeline which joins a $125 \mathrm{~Hz}$ ABP signal with $500 \mathrm{~Hz}$ ECG signal based on their timestamps. First, the small gaps in both waveforms are filled using signal value imputation. Next, the ABP signal is upsampled to match the frequency of ECG. Finally, the signal values are normalized before joining them together to pair up strictly overlapping events. Even

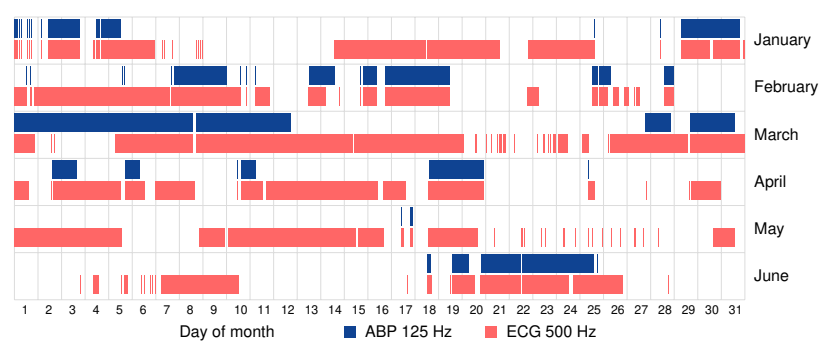

Figure 2: Distribution of ECG and ABP signals collected from a single monitoring device over first six months of 2019

though there are several general-purpose solutions [3, 10, 11, 54, 59] proposed in the past to build and process such data flow pipelines, from our experience closely working with the clinicians, data analysts, and machine learning researchers at The Hospital for Sick Children, we recognize several new challenges that make physiological waveform data processing unique.

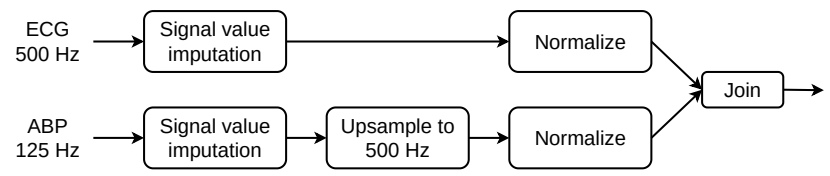

Figure 3: A sample data processing pipeline on ECG and ABP

First, the choice of operations and transformations applied on the waveform data varies considerably based on use cases. Therefore, the data scientists should have the freedom to experiment with different data processing pipelines and should be able to do so with minimal effort. Moreover, most of the operations and transformations applied to the waveform data follow a strict notion of the data's temporal ordering. This necessitates a flexible and easy to use programming interface with in-built temporal logic support. Second, the data analysts and ML researchers usually first perform the experiments and analysis on the retrospective (i.e. historical) data stored in the persistent disks and then deploy their solutions on real-time data once the algorithms are finalized. The deployment must be seamless and error-free.

Finally, since the physiological data collected are fully-identified, there are several legal restrictions [19] in-place on moving the data outside hospital facilities. Even though there have been recent efforts to de-identify data for public research purposes (e.g., MIMIC [25]) or propose data analytics systems with strong security guarantees (e.g., Opaque [60]), most hospitals still prefer to keep their data private, citing the high risk associated with the leakage of patient information [1]. Hence, the physiological data processing systems has to perform computations within limited hardware budget available in hospitals and still provide high performance.

\section{TEMPORAL STREAM PROCESSING FOR PHYSIOLOGICAL DATA ANALYSIS}

Since physiological waveform data is produced in a streaming fashion, stream processing [6] is a natural choice for building aforementioned data processing pipelines. Most of the modern streaming 


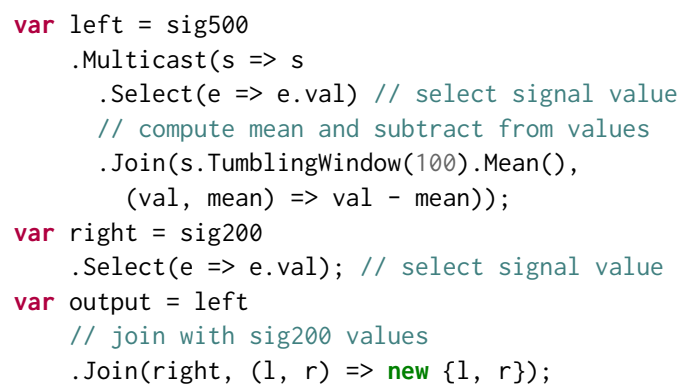

\section{Listing 1: Running example of a temporal query}

engines [3, 5, 11, 54, 59] support some temporal query language with an implicit notion of event time, temporal ordering, and finegrained windowing strategies. To illustrate, Listing 1 shows the query for a simplified version of the pipeline in Figure 3 which joins a $500 \mathrm{~Hz}$ signal (sig500) and $200 \mathrm{~Hz}$ signal (sig200) after a series of transformations. ${ }^{2}$ First, the signal values of sig500 is adjusted by taking the mean of signal values on $100 \mathrm{~ms}$ TumblingWindow (fixed-size, non-overlapping, and contiguous intervals) and subtracting that from the original values. These transformed signal values are joined with the signal values from sig200 using temporal Inner foin. Modern streaming engines provide a simple and flexible programming interface for writing complex data processing pipelines through such query languages. However, most contemporary streaming engines are built with distributed setup in mind and, unfortunately, exhibit sub-optimal single machine performance due to low hardware utilization $[11,27]$. At the same time, streaming engines that are optimized for single machine performance (e.g., Trill [11]) demonstrate orders of magnitude higher performance.

To validate this in the context of waveform data processing, we compare the single-core performance of the temporal foin operation in four major state-of-the-art streaming engines: (i) Spark streaming [5, 59], (ii) Flink [10], (iii) Storm [54], and (iv) Trill [11]. foin is one of the most commonly used primitive operations in physiological data processing. Finding the temporal correlation of different signal streams or computing derived variables such as aggregates and joining them back with the input stream events are frequently performed computations. Therefore, the performance of foin operation can considerably affect the entire pipeline's performance in such scenarios.

Table 1 shows the number of signal events joined per second by different streaming engines. We observe that Trill outperforms other streaming engines by more than $10 \times$. Trill's performance benefits come from a better memory management system, improved cache locality using columnar data representation, and the use of hand-optimized primitive operators.

Unfortunately, despite all these optimizations that made Trill significantly better than its competitors, we observe that the performance of Trill is far from being competitive with the hand-tuned implementations used by the data scientists. Such implementations are usually based on numerical libraries such as SciPy [38], NumPy [37], and Scikit-learn [42] and provide a rich ecosystem

\footnotetext{
${ }^{2}$ We use signal frequencies $500 \mathrm{~Hz}$ and $200 \mathrm{~Hz}$ to show that LifeStream can handle misaligned signals as well.
}

Table 1: Throughput of Spark, Storm, Flink, Trill, and SciPy (in million events/sec)

\begin{tabular}{c|ccccc}
\hline Benchmarks & Spark & Storm & Flink & Trill & SciPy \\
\hline Temporal Join & 0.07 & 0.04 & 0.09 & 0.80 & - \\
Upsampling & - & - & - & 0.69 & 15.06 \\
\hline
\end{tabular}

of highly efficient data processing operators. Table 1 shows the performance comparison of signal upsampling [58] operation implemented in Trill and the corresponding implementation available in the SciPy library. We observe that Trill is about $22 \times$ slower than SciPy. This makes numerical libraries seem like a better choice for building data processing pipelines from a performance perspective. Even though this is the status quo among data scientists, we argue that such an approach has significant drawbacks from a programmability and system maintainability perspective because of the following reasons.

First, the lack of implicit notion of event time and support for flexible windowing strategies make building physiological data processing pipelines using numerical libraries significantly harder and more complicated for data scientists. For instance, writing the data transformations in Listing 1 would require data scientists to manually maintain the temporal ordering of the data at the application level. Moreover, making simple tweaks like modifying the pipeline to use a rolling mean would only take a single line of change from TumblingWindow to SlidingWindow in a temporal query language. The same change would require a complete redesign of the code base in the typical numerical library-based approaches.

These limitations force data scientists to make one of two undesirable choices. (i) To put considerable engineering effort to implement temporal features on top of the numerical libraries, or (ii) make compromises in their experimentation and requirements to adjust to the restrictions imposed by numeric libraries. Secondly, a lack of common API specifications and data abstractions across different libraries further complicates building large data processing pipelines, as the data scientists need to make sure the correctness of the input data types of each function and additionally perform type conversions when necessary. Such an approach quickly becomes unmanageable and error-prone as the data pipeline gets larger and more complex.

Despite all these drawbacks, data scientists still choose to go with such adhoc library-based methods for building data processing pipelines instead of more systematic approaches (e.g, using stream processing engines such as Trill) due to the significant performance benefits associated with numerical libraries. As a result, we observe that data scientists spend most of their development time writing peripheral code and extra "glue" logic to wire different numerical libraries together [48], instead of focusing on their primary goalanalysing data and generating insights from it.

On the other hand, the library-based approach seems desirable from the performance perspective at first. However, prior works [39-41] have pointed out that even though the individual functions in these libraries may achieve high performance in isolation, they usually fail to maintain those benefits in a more complex workflow with a combination of functions because of the overhead 


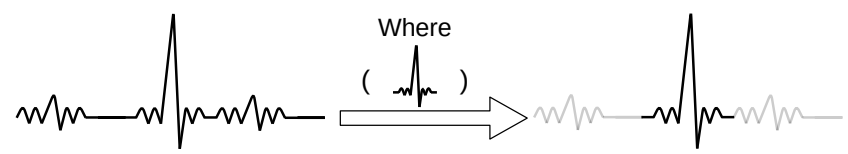

Figure 4: Shape detection using extended Where query

associated with intermediate data conversion and lack of cross function optimizations. This makes numerical libraries a poor choice for building physiological data processing pipelines even from a performance standpoint.

Based on the above observations, we conclude that a physiological data processing system must provide a programming interface similar to the ones supported by the major streaming engines with flexible windowing strategies and an implicit support for event time and temporal ordering of the data. Moreover, the system must efficiently utilize the available hardware resources to provide high performance.

\section{LIFESTREAM: SYSTEM OVERVIEW}

To address the challenges described in the previous section, we introduce LifeStream, a temporal query processing engine specifically optimized for physiological waveform data processing. Compared to alternative approaches [11, 37, 38, 42, 59], LifeStream provides: (i) superior performance by taking advantage of the periodic nature of the waveform data and optimizing the end-to-end pipeline, (ii) a rich temporal query language support ${ }^{3}$ with simple and flexible primitive temporal operations and fine-grained windowing support, and (iii) several extensions on traditional temporal operations that are useful for physiological data processing such as extending Where operator to query visual patterns and shapes in data streams as shown in Figure 4 (see Section 6.1 for more details).

LifeStream provides the data abstraction that consists of a stream of events in chronological order. An event is a single unit of data with three fields: (i) a user-defined payload, (ii) a sync time, which dictates the time from which that particular event is active, and (iii) a duration, which defines the active lifetime of the event. LifeStream is exclusively targeting data streams in which events appear at constant intervals. That means the sync time of every event is always at the period boundaries. Since each event's position in a stream is predictable, we use the symbolic representation of (offset, period) to describe a stream, where offset is the sync time of the first event in the stream and period is the reciprocal of the frequency. Even though we are primarily targeting physiological waveform data, any streaming data that can be represented in the aforementioned format can take advantage of the benefits of LifeStream. This includes periodic streaming datasets such as performance counters produced in data centers [18], data collected from wearable devices [17], and real-time sensor data [36].

One of the key design choices we make in LifeStream is to decouple the operator implementation from the data representation used for storing stream data. Most of the streaming engines, such as Trill [11] and Spark streaming [5] implement their operators to take an arbitrary sequence of events from the input stream data in the form of a batch, and produce another batch as its output. In our

\footnotetext{
${ }^{3}$ Similar to the one provided by Trill.
}

work, we realize that this approach has several severe limitations. First, the locality of the computation is highly tied with the batch size. Therefore, usually, the system has to trade-off the benefits of large batch processing in favor of preserving locality. Second, we observe that such operator implementations limit different compiletime and runtime optimizations we can perform on LifeStream (see Section 5 for more details).

To avoid such limitations, we introduce a new key construct called fixed interval sliding window or FWindow. FWindow is an arbitrary interval within a stream. Similar to an event, FWindow also has a sync time corresponding to the starting timestamp of the interval and a fixed size or duration. Since FWindow is an interval on a stream, the size of the FWindow should always be a multiple of the period of the stream. In LifeStream, we implement all the operations based on FWindows-the operators typically take one or two FWindows as input and produce a single FWindow as output. Operators can slide the FWindows to read different parts of the stream at runtime by updating its sync time. The only restriction is that FWindows can only move forward in time to ensure monotonic progress in query execution.

Table 2 describes the set of primitive operations supported in LifeStream with which data scientists can write queries on streaming data. The query is compiled into a computation graph composed of FWindows and temporal operators. The size of the FWindows are initially set to the same value as the corresponding stream's period. Figure 6(a) shows the initial computation graph prepared from the example query in Listing 1 . The FWindows are represented using a symbolic representation (offset, period)[dimension] where the dimension is the FWindow size. This graph is then passed on through a graph transformation process to generate the final executable computation graph. Finally, the input data is streamed through the executable computation graph to generate the result.

\section{LIFESTREAM: KEY OPTIMIZATIONS}

LifeStream maximizes resource utilization by (i) improving cache locality, (ii) reducing runtime overhead, and (iii) pruning redundant computations. We achieve this goal by identifying two key properties of the temporal operations on periodic streams described in Section 5.1. Using these properties, we propose three major query compilation and execution time optimizations described in Sections 5.2 and 5.3 .

\subsection{Properties of Temporal Operations on Periodic Streams}

Linearity of temporal operations: The sync time of events in the output stream of a temporal operator is a linear transformation of that of the input events.

This property allows LifeStream to map every output event of a temporal operator to the corresponding parent input event(s). Figure 5 shows how the event times change in the output stream of several common temporal operators such as Select, Shift, and foin when applied on a periodic stream. One consequence of this property is that the period and the offset of the output stream is also a linear transformation of that of the input stream and can be computed statically. Moreover, this allows to map the events in the output stream of an operator to the corresponding parent 
Table 2: Primitive temporal operations supported by LifeStream

\begin{tabular}{|c|c|c|c|}
\hline Operation & Dimension & Is stateful? & Description \\
\hline Select & {$[\mathrm{out}] \leftarrow[\mathrm{in}]$} & No & Performs a projection on the payloads of the stream events. \\
\hline Where & {$[$ out $] \leftarrow[$ in $]$} & No & Filter out events based on a predicate. \\
\hline Aggregate(w, p) & {$[$ out $] \leftarrow p$} & No, if $w=p$ & $\begin{array}{l}\text { Applies a user-defined aggregate function to } w \text {-sized win- } \\
\text { dows with stride length of } p \text {. E.g., Sum, Max, and Mean. }\end{array}$ \\
\hline Join & {$[$ out $] \leftarrow L C M([$ left $],[$ right $])$} & $\mathrm{No}^{*}(\mathrm{Sec} 6.3)$ & $\begin{array}{l}\text { Performs temporal equijoin between two streams. E.g., In- } \\
\text { nerJoin, LeftJoin, and OuterJoin. }\end{array}$ \\
\hline ClipJoin & {$[$ out $] \leftarrow[$ in $]$} & Yes & $\begin{array}{l}\text { Joins each event in one stream with immediately succeeding } \\
\text { event in another. }\end{array}$ \\
\hline Chop & {$[$ out $] \leftarrow[$ in $]$} & Yes & $\begin{array}{l}\text { Splits the intervals of each event on user-defined period } \\
\text { boundaries. }\end{array}$ \\
\hline Shift(k) & {$[$ out $] \leftarrow[$ in $]$} & Yes & Shifts the sync time of each event by a constant. \\
\hline AlterPeriod(p) & {$[$ out $] \leftarrow[\mathrm{in}]$} & No & Alters the period of a stream. \\
\hline AlterDuration & {$[$ out $] \leftarrow[\mathrm{in}]$} & No & Alters the duration of each event in the stream. \\
\hline Multicast & {$[$ out $] \leftarrow[$ in $]$} & No & $\begin{array}{l}\text { Forks a stream to allow multiply subqueries to be performed } \\
\text { on same input stream. }\end{array}$ \\
\hline Transform(w) & {$[$ out $] \leftarrow w$} & No & $\begin{array}{l}\text { Applies a user-defined transformation function on } w \text {-sized } \\
\text { windows that produces another } w \text {-sized window as output. }\end{array}$ \\
\hline
\end{tabular}

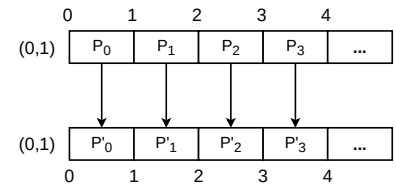

(a) Select

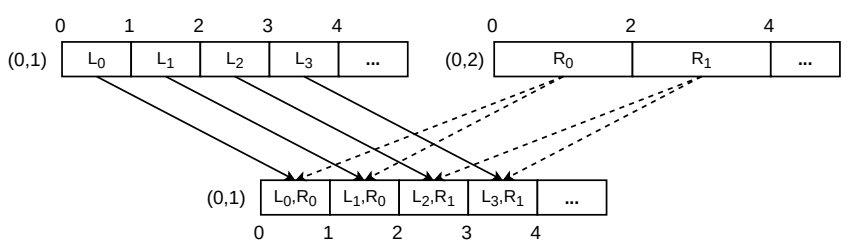

(c) Join

Figure 5: Event lineage tracking

events in the input stream(s). Figure 5 shows how the output events are mapped to the input events after an operator transformation. Since all temporal operators in LifeStream follow this property, the mapping can be extended from the final output stream events of the query all the way to the initial input stream events. We call this mechanism event lineage tracking and use in LifeStream to improve both the cache locality and prune redundant computations.

Bounded space complexity: For a stream with period $p$, the maximum number of events that can be present within a given time interval $d$ is bounded by $O(d / p)$.

One of the key properties of a periodic stream is that two events in a stream can not overlap with each other, which means there can only be at most one event active at any point in time within a stream. Therefore, the maximum number of events in an interval is bounded by the duration of that interval. Moreover, since temporal operations also follow linearity property, all the intermediate streams generated in the query should also be periodic, and thus should also satisfy bounded space complexity property. LifeStream uses this observation to estimate the maximum memory footprint of all the intermediate results and preallocate them to minimize the runtime memory allocation and deallocation overhead commonly observed in other streaming engines [11, 43].

\subsection{Locality Tracing and Memory Footprint Estimation}

One thing that makes stream processing attractive is that even though the data it processes is usually massive (and sometimes can even be potentially infinite), the computations performed on the data are highly local and require only to deal with a small continuous window of events within the stream. Most streaming engines take advantage of this locality property only at an individual operation-level and do not optimize or even maintain crossoperation locality. In LifeStream, we introduce a method called locality tracing that uses periodic streams' linearity property to precisely estimate the end-to-end locality of the computations in the entire pipeline. Locality tracing performs static analysis on top of the computation graph and adjusts the dimensions of all the FWindows to make sure that the input and output dimensions of all the operators match. Table 2 describes the dimension translation of each supported operation in LifeStream.

Figure 6 shows the locality tracing procedure performed on the example query in Listing 1 . The procedure starts from the end of the pipeline, and LifeStream identifies a mismatch in the last foin operation's $\left(\mathrm{Join}_{2}\right)$ input and output dimensions. Since the FWindow sizes has to be a constant multiple of the periods, to match the dimensions of $\mathrm{Join}_{2}$, LifeStream sets the FWindow sizes to the least common multiple (LCM) of the input and output dimensions. In this case, the dimensions are set to 10 . Next, the dimensions of the $J_{o i n}$ operation are adjusted similarly. However, this adjustment 


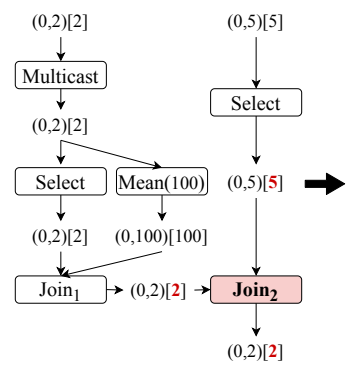

(a) Initial computation graph

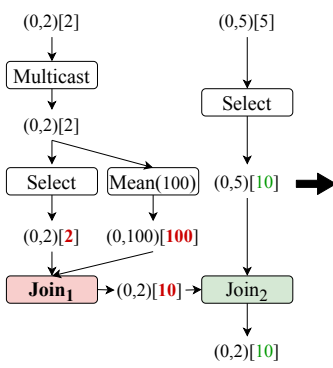

(b) Adjust $\mathrm{Join}_{2}$

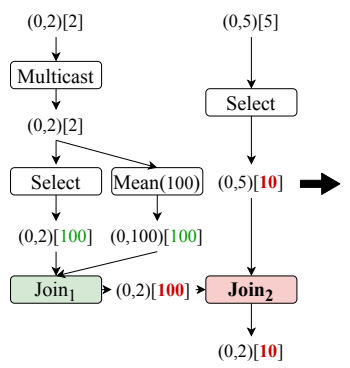

(c) Adjust Join 1

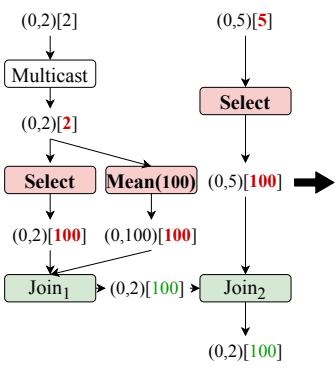

(d) Re-adjust $\mathrm{Join}_{2}$

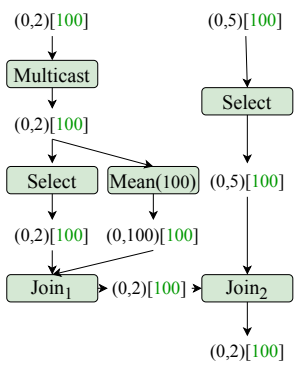

(e) Final computation graph

Figure 6: Locality tracing procedure on the example query

introduces a mismatch in $\mathrm{Join}_{2}$, which is corrected in the next step. This procedure is continued until all operations have uniform input and output dimensions. This graph transformation ensures that the intermediate results are consumed immediately by the subsequent operation(s), which, in turn, maximizes the locality of the end-to-end query.

Once the dimensions of all the operations are computed, LifeStream uses the bounded space complexity property to determine the maximum memory footprint of all the FWindows. LifeStream then preallocates this memory statically and keeps reusing the same memory during runtime to minimize dynamic memory allocation overhead, commonly observed in other streaming engines [43].

\subsection{Targeted Query Processing}

Most streaming engines process queries in an eager fashion where the query computation is initiated at the data ingestion side, and each subsequent operators perform the corresponding transformations on the input as soon as it receives the data and immediately pass it on to the next operator down the pipeline, irrespective of whether the next operation would need to process that data or not. In physiological data processing, this introduces many redundant computations as the data contains a high degree of discontinuity.

One of the most common examples would be the use of Inner foins to match overlapping events of multiple signal streams after a series of compute intensive data transformations (Figure 3). Figure 2 shows that the mutually overlapping regions in ECG and ABP signal streams are far fewer than the total number of events in the individual streams. In an eager query processing model, all the events from both streams are invariably going to be passed through the intermediate transformations, even though most of them are eventually going to get discarded by the final foin operation.

In LifeStream, we address this issue by introducing a runtime optimization called targeted query processing. At runtime, LifeStream uses event lineage tracking to map output FWindows of the endto-end pipeline to corresponding parent FWindows in the input stream(s). This lets LifeStream selectively target regions of the input stream(s) by sliding the FWindows and running the computations only when an output FWindow is expected to produce. Hence, targeted query processing lets LifeStream skip all those computeheavy transformations in the presence of discontinuities in the input data stream and focus only on the relevant parts of the data.

\section{LIFESTREAM: IMPLEMENTATION}

We implement LifeStream as a library using C\# programming language and .Net core v3.1 framework [30]. Hence users of LifeStream can also benefit from high-level language features such as arbitrary data types, integration with custom program logic, and a rich library ecosystem [31]. Users can also create streams from various sources, including real time data through networks, retrospective data from files, and cached data from the main memory.

As described in Section 4, the key building block that temporal operators use to access streaming data is FWindow. In FWindow, events are indexed by their sync time. In addition to the event payload, FWindow contains three extra fields: vsync, duration, and bitvector. Vsync and duration fields store the sync time and duration of the events. Bitvector is used to mark the absence of an event. Every event in the FWindow has an associated bit that can either be 0 or 1 to indicate the event's presence or absence. All the fields in the FWindow are stored in columnar format in order to maximize cache locality as most operators only need to read from or write to a subset of the fields.

Below, we describe the details about the extended temporal query language supported in LifeStream and a few implementation challenges we faced and corresponding solutions.

\subsection{Temporal Query Language Extensions}

Apart from performance benefits, LifeStream also provides several additional important features through the query language extensions. We introduce a generic Transform primitive operation which lets users write arbitrary transformations on a fixed interval of events. This operation helps users to integrate third-party libraries into the stream processing pipeline easily.

We also extend the Where query primitive operation to support shape-based querying. As shown in Figure 4, users can input arbitrary artifacts or patterns they want to detect in the stream as a list of signal values. We use a variation of the dynamic time warping (DTW) algorithm [56] called constrained DTW [33] and re-purpose it for streaming scenario to only compute the DTW distance in linear time.

Figure 7 shows an artifact commonly found in the arterial blood pressure (ABP) signal called line-zero, which occurs when the pressure sensors attached to patients are calibrated against atmospheric 


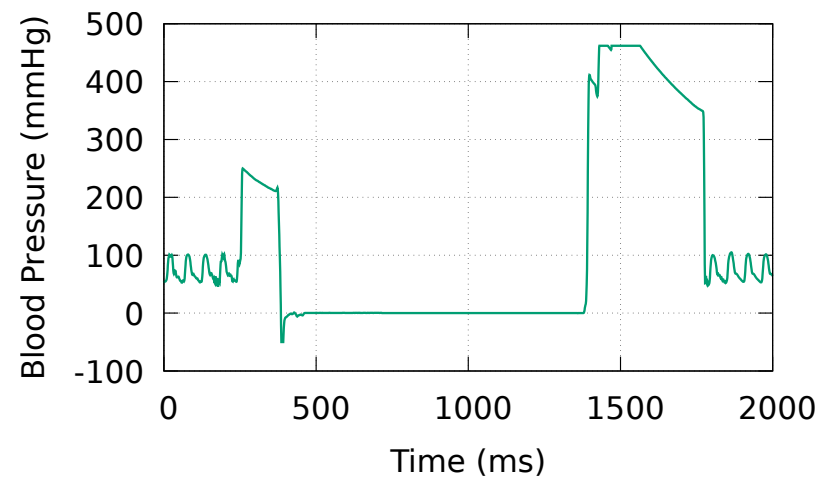

Figure 7: Line-zero artifact in arterial blood pressure (ABP)

pressure. In such cases, the $\mathrm{ABP}$ signal values produced by the monitors would show this characteristic shape because of the distortion in the measurements. Several other artifacts are found in different signals, and data scientists generally want to remove such regions from the physiological datasets as this could negatively affect their data analysis process.

In LifeStream, data scientists can use the extended Where query primitive to filter out these artifacts from the stream by providing a representative shape as input to the query in the form of a sequence of signal values. LifeStream subsequently uses the re-purposed DTW algorithm to do the pattern matching in the input stream. We measure the algorithm's accuracy over a month of ABP signal data from a single device containing 49 line-zero artifact and achieve $0 \%$ false negatives and $0.2 \%$ false positives. This shows that LifeStream can accurately detect such characteristic shapes in the data streams.

\subsection{FWindow Fragmentation}

Since LifeStream stores and accesses data at the granularity of FWindows and they contain events of continuous fixed size interval, having small gaps in the input data might cause memory fragmentation. This might lead to low memory utilization, and in turn, low query execution performance. However, as shown in Figure 2, most of the raw physiological data's discontinuities are generally concentrated on specific time periods rather than being randomly scattered throughout the stream. Hence most parts of the stream has continuous and densely packed sequence of data which ensures high memory utilization. However, when small gaps occur, we handle them by setting the bitvector field in the corresponding position in the FWindow to mark the absence of the events.

Another possible cause of fragmentation is when Where query is used to filter out certain events in the stream, based on an arbitrary predicate defined by the programmer. From our experience building pipelines using LifeStream, Where operation is the least commonly used primitive operation. Even when used, it is mostly to filter out a large continuous portion of the stream (e.g., remove noisy regions or artifacts from the data). Therefore, the chances for FWindow fragmentation are minimum.

In the use cases that we evaluate in this work (see Section 8), we observe the degree of the FWindow fragmentation is at most $0.3 \%$, which is too small to impact query performance negatively.

\subsection{Stateful Temporal Operators}

Since the query execution in LifeStream is done at the FWindow granularity and the memory allocated for the FWindows is rewritten, certain operators may need to maintain an internal state throughout the query execution (e.g., rolling aggregate operations). To handle such cases, LifeStream allows operators to create constant size states during initialization, to preserve bounded memory property and ensure that there is no dynamic memory allocation during operator execution.

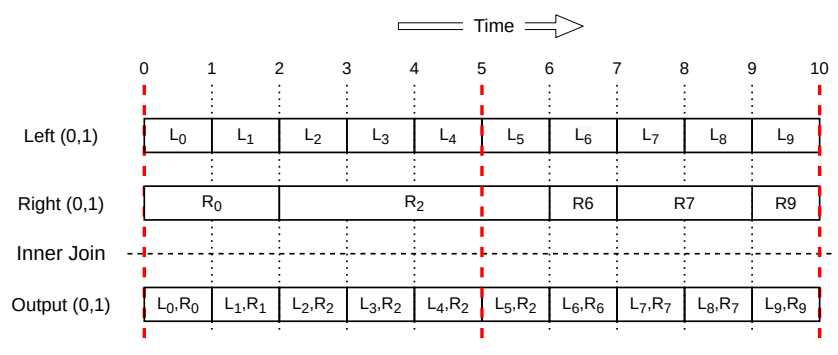

Figure 8: Stateful Join operation

Another case of a stateful operator is shown in Figure 8. In this case, a constant duration stream (Left) is inner joined with another stream with arbitrary duration (Right). The red dotted lines represent the FWindow boundaries. As shown in the figure, event $R_{2}$ in the Right stream has overlapping events in the Left stream in both FWindows. In such cases, in order for LifeStream to produce the output event $\left(L_{5}, R_{2}\right)$ correctly, the Inner foin operation needs to save the event $R_{2}$ in its state before moving to the second FWindow. However, the periodic nature of these streams ensures that there can only be at most one such event in a stream that can cross the interval boundary of the FWindow at any point in query execution. Therefore, the state required for temporal Join is always constant, and stateful foin operators preserve the bounded memory property.

\section{METHODOLOGY}

Benchmarks: We evaluate LifeStream on three categories of benchmarks. (i) Primitive micro-benchmarks: This benchmark category includes several primitive temporal operations like Select, Where, Aggregate and temporal Inner foin. (ii) Operation benchmarks: This includes five operations described in Table 3 that we find commonly used by the data analysts to process physiological data. (iii) End-to-end applications: We use the data processing pipeline described in Figure 3 using the operations in Table 3 as our primary benchmark for the end-to-end performance evaluation. On top of this, in Section 8.4, we also use two additional pipelines used in real-world data analysis in order to show both the performance and generality of LifeStream.

Datasets: Physiological waveform data we use contains signal events with a 64-bit timestamp and 32-bit floating point value. For the experiments, we use two dataset types. (i) Synthetic data: $1000 \mathrm{~Hz}$ waveform data generated for 1000 minutes with randomly selected signal values. This dataset contains a continuous stream of signal events with no gaps. (ii) Real data: A private dataset from The Hospital for Sick Children, containing physiological waveform data collected from 6100 patients over the past five years. The dataset 
Table 3: Operation benchmarks and their descriptions

\begin{tabular}{lll}
\hline Operation & Libraries & Description \\
\hline \hline Normalize & $\begin{array}{l}\text { Scikit- } \\
\text { learn }\end{array}$ & $\begin{array}{l}\text { Normalize a window of signal values using } \\
\text { standard scores. }\end{array}$ \\
\hline PassFilter & SciPy & $\begin{array}{l}\text { Filter frequencies using finite impulse re- } \\
\text { sponse [46]. }\end{array}$ \\
\hline FillConst & NumPy & $\begin{array}{l}\text { Fill gaps smaller than the given window size } \\
\text { with a constant value. }\end{array}$ \\
\hline Resample & SciPy & $\begin{array}{l}\text { Fill gaps smaller than the given window size } \\
\text { with the mean of the values in the window. }\end{array}$ \\
& & $\begin{array}{l}\text { Upown sample the signal using linear in- } \\
\text { terpolation [58]. }\end{array}$ \\
\hline
\end{tabular}

contains more than 830,000 patient-hours of data and 250 different signal types. However, for our experiments, we only use arterial blood pressure (ABP) and electrocardiogram (ECG) signals sampled at their default rate $125 \mathrm{~Hz}$ and $500 \mathrm{~Hz}$ respectively [16].

Baselines: We compare the performance of LifeStream with two baselines. (i) Microsoft Trill, a state-of-the-art temporal query processing engine specifically optimized for single machine performance. (ii) Numeric libraries (NumLib) like SciPy, NumPy and Scikitlearn with hand-optimized implementations for data processing operations. For end-to-end benchmarking, we implement the numerical library-based data processing pipeline in Python. To make fair performance comparisons, we tried to minimize computations done on native Python as much as possible by offloading the heavy processing to the numerical library functions. However, operations like temporal Inner foin required pure Python implementation.

Metrics: We use the total execution time from a single core on a fixed input data size as the primary comparison metric for performance on primitive micro-benchmarks, operation benchmarks, and end-to-end applications. For scalability experiments, we use the throughput obtained on multiple cores/machines. Throughput is measured as the average number of signal events processed per unit time. Both execution time and throughput reported is the average of measurements from 10 trials. The standard deviation of the measurements are observed to be less than $1 \%$. For the sensitivity study on cache utilization, we use total number of last level cache (LLC) misses (median over 5 trials) on fixed workload as a comparison metric measured using Intel vTune profiler v2020 [24].

For all the experiments except scalability, we use 8-core (16 hyperthreaded) Intel Xeon CPU E5-2660 machine running at $2.2 \mathrm{GHz}$, with 16 GB RAM, and running 64-bit Ubuntu 20.04. For scalability experiments, we use up to 16 AWS EC2 m5a.8xlarge [4] machines each with 32 cores and 128 GB DRAM. We use a window size of 1 minute for all the benchmarks unless otherwise specified.

\section{EVALUATION}

We evaluate LifeStream to answer the following questions:

(1) How does the performance of LifeStream compare to stateof-the-art streaming engines and numerical libraries?

(2) Can LifeStream accelerate end-to-end performance of physiological waveform data processing pipelines?

(3) How beneficial are the proposed optimizations?

(4) How well does LifeStream scale on multiple machines?

(5) Can LifeStream handle diverse data analytics requirements?

\subsection{Primitive Micro-benchmarks}

We compare the performance of LifeStream against Trill on 7 most commonly used primitive temporal operations using the synthetic dataset for these experiments. Figure 9(a) shows the execution time taken by both Trill and LifeStream and based on the results, we make the following two major conclusions.

First, on simple operations such as Select and Where, performance of LifeStream is within $20 \%$ of that of Trill. This shows that LifeStream is not adding any significant overhead over already highly optimized operations in Trill. Second, we observe that LifeStream shows much higher performance benefits as the operations become more complex. Operators such as Aggregate, Chop, Clipfoin, and foin are respectively $2.17 \times, 1.98 \times, 5.34 \times$, and $6.65 \times$ faster than its Trill counterparts.

We attribute LifeStream's high performance on primitive operations to the introduction of FWindow. FWindow greatly simplifies the operator implementations and eliminates the need for using complex data structures such as hashmaps in temporal foin like Trill. Moreover, since the events' timestamps and index positions are aligned, operators implemented in LifeStream can compute each event's sync time from its index position without having to read from memory. Such implementation-level optimization makes LifeStream efficient even at the primitive operation-level.

\subsection{Operation Benchmarks}

To evaluate the performance of common physiological data transformations, we implement the operations listed in Table 3 on LifeStream by writing queries using the temporal operators and compare their performance against the similar queries written in Trill and the hand-tuned implementations available in the corresponding numerical libraries specified in Table 3. We conduct this experiment on a $500 \mathrm{~Hz}$ ECG signal from the real dataset containing $126 \mathrm{M}$ events. Figure 9(b) shows the execution time of Trill, numerical libraries (NumLib), and LifeStream on each benchmark. We make three major conclusions from this figure.

First, across all the operations, LifeStream is shown to be 5 $11.21 \times$ faster than Trill. This shows the effectiveness of the optimizations implemented in LifeStream. Second, LifeStream also exhibits comparable performance against highly optimized implementations available in the numerical libraries (within $50 \%$ performance of the popular numerical libraries we evaluate). Third, in some instances such as a very commonly used Normalize operation, LifeStream even surpasses the hand-tuned performance provided by Scikit-learn library by $1.35 \times$. This asserts our claim that LifeStream provides ease of programming of a temporal query language without sacrificing performance.

\subsection{End-to-End Applications}

To evaluate whether LifeStream can improve the end-to-end performance of data processing, we build the pipeline shown in Figure 3 over LifeStream, Trill, and in Python using numerical libraries (NumLib). The data pipeline in all three implementations process $500 \mathrm{~Hz}$ ECG and $125 \mathrm{~Hz}$ ABP signals from the real dataset stored in CSV format and produces a joined signal stream after running a series of transformations on the data shown in Figure 3. The dataset contains two weeks of data from a single monitoring device with 

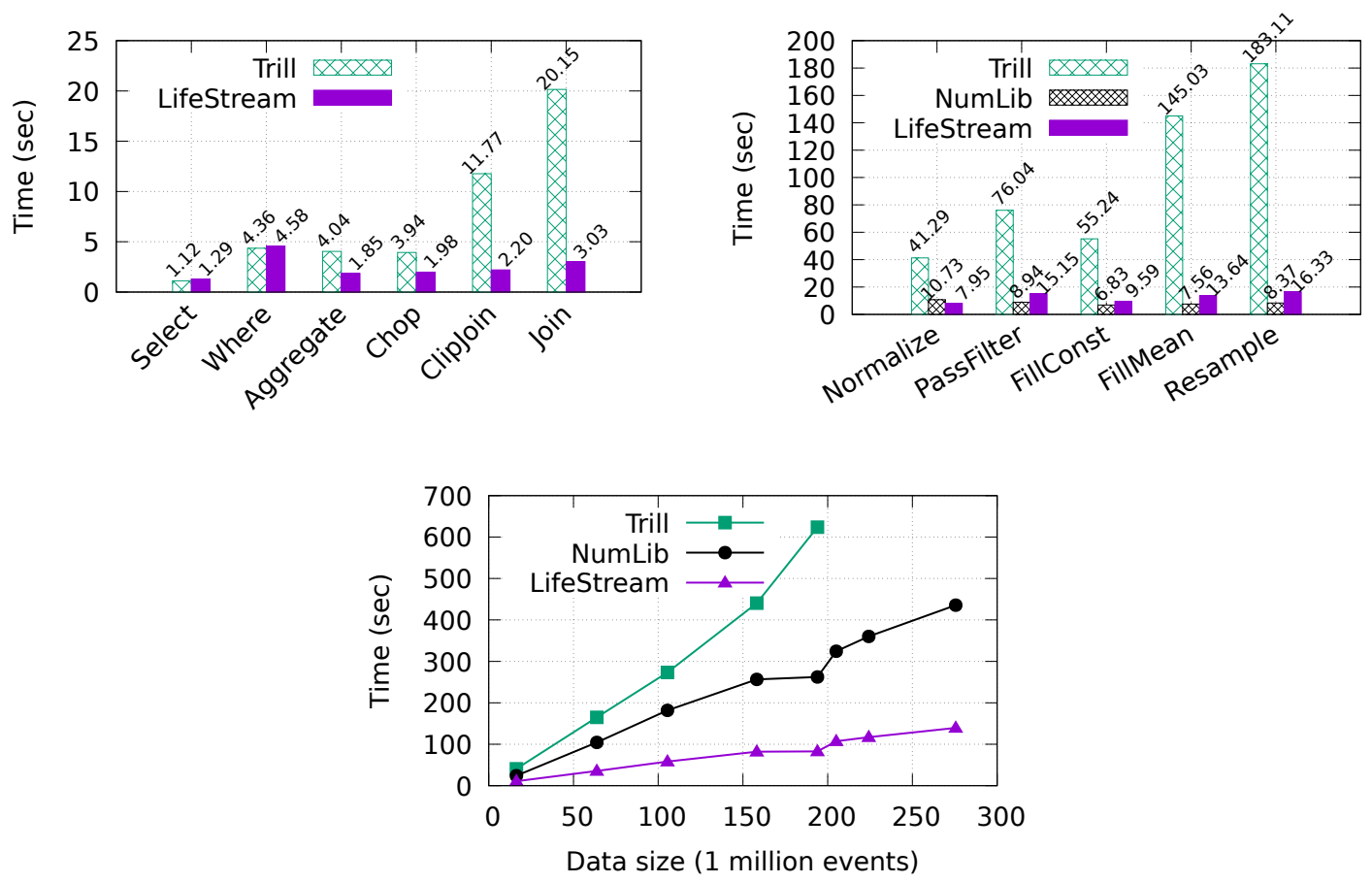

Figure 9: (a) Primitive micro-benchmarks, (b) Operation benchmarks, (c) End-to-end applications

$275 M$ signal events. Figure 9(c) shows the end-to-end execution time by varying the dataset size for all three implementations. We make the following two major observations from this figure.

First, LifeStream outperforms both Trill and NumLib by $7.5 \times$ and $3.2 \times$ respectively. This reinstates that, even though individual operators in the numerical libraries can exhibit high performance when executed in isolation (as we show in Section 3), it does not necessarily translate into the best end-to-end performance due to data conversion overhead and lack of end-to-end optimizations [40].

Second, in the case of Trill, as the size of the dataset increases, the execution time rises rapidly, and Trill goes out of memory at $200 M$ events. Our investigation reveals that this happens because of Trill's implementation issue with the foin operation. Trill expects both left and right streams of the Join operation to progress at a similar pace. However, if the two streams diverge considerably, the Join operator's internal memory keeps accumulating until the available memory is exhausted. Since physiological data contains a high degree of discontinuity, it is very common for such divergence to occur during query processing. LifeStream uses targeted query processing optimization (described in Section 5.3) to skip over such non-overlapping parts of the input data.

\subsection{Generality}

Using LifeStream, we were able to bring two data analytics models developed at the Sickkids hospital into practical deployment and real-world use, namely line-zero artifact detection model (LineZero) and cardiac arrest prediction model (CAP) [52]. These models demand diverse data analytic requirements. For instance, line-zero artifact detection model performs a sliding window-based normalization operation. The cardiac arrest prediction model pipeline joins 6 different signal types together after performing operations like normalization, signal upsampling, signal value imputation, and event masking on each signal stream.

\section{Table 4: Throughput in million events per second}

\begin{tabular}{c|cc|c}
\hline Model & Trill & LifeStream & Speedup \\
\hline LineZero & 0.027 & 0.315 & $11.58 \times$ \\
CAP & 0.174 & 0.877 & $5.04 \times$ \\
\hline
\end{tabular}

Table 4 shows the single thread performance of LifeStream over Trill on LineZero and CAP models. Like the end-to-end benchmark results, LifeStream achieves $11.58 \times$ and $5.04 \times$ speedup over Trill on LineZero and CAP respectively.

\subsection{Sensitivity Studies}

In this section, we conduct experiments to analyze the effectiveness of the optimizations applied in LifeStream.

Cache Utilization. To analyze how well LifeStream utilizes the cache compared to Trill using optimizations such as locality tracing, we conduct an experiment to measure the last level cache (LLC) misses of both engines on one of the most commonly used operation - Normalize. In order to avoid the influence of data discontinuities in the measurements, we use synthetic dataset for this experiment. We use Intel vTune [24] to measure the cache misses during the query execution over a constant size input dataset. 
Table 5: The number of last level cache misses (in millions)

\begin{tabular}{c|ccc}
\hline Batch size & $10^{5}$ & $10^{6}$ & $10^{7}$ \\
\hline Trill & 2.43 & 4.11 & 6.73 \\
LifeStream & 0.79 & 0.82 & 0.96 \\
\hline
\end{tabular}

Table 5 shows the LLC misses in both Trill and LifeStream on three different batch sizes. For a batch size of $10^{5}$, LifeStream is experiencing $3 \times$ lower cache misses as that of Trill. As the batch size increases, the number of cache misses in Trill increases significantly while LifeStream's miss rate stays relatively constant. As we describe in Section 5.2, Trill's inability to preserve cross-operation locality is the primary reason for this behaviour. The consequence of this limitation becomes more pronounced on larger batch sizes. LifeStream, on the other hand, preserves the end-to-end locality of the query using locality tracing irrespective of the batch size.

Targeted Query Processing. This section analyzes the effectiveness of targeted query processing while running large data processing pipelines. We pick the ECG and ABP signals of several different dates from the real dataset with varying degrees of overlapping events between them to perform this analysis. Figure 10(a) shows the relative performance speedup of LifeStream over Trill, measured with respect to the percentage of overlapping events in these data subsets. We observe that, as expected, the speedup is smaller when there is near perfect overlap, which is about $7 \times$. The speedup starts to increase as the degree of overlap decreases, because LifeStream can skip more and more redundant computations compared to Trill. For example, a day with $10 \%$ overlap in ECG and ABP leads to about $38 \times$ speedup over Trill, which is about $5 \times$ higher than the base performance of LifeStream.

Window size In this section, we conduct a sensitivity study on LifeStream to measure the effect of window size on its performance. Figure 10(b) shows the execution time of Trill and LifeStream on the end-to-end benchmark over the synthetic dataset with window size varying from 1 minute to 1 hour. The results suggest that LifeStream can maintain its performance benefits compared to Trill even on larger windows.

\subsection{Scalability}

Physiological datasets generally contain signals collected from thousands of patients, and the data processing pipelines usually process data from different patients separately. That means the data processing can be parallelized across multiple patients. LifeStream takes advantage of this data-parallel nature of the physiological dataset to scale up the computation to both (i) multiple cores within a machine and (ii) multiple machines.

We evaluate the scalability of LifeStream and compare it against Trill and numerical libraries on a single AWS m5a.8xlarge [4] machine with 32 cores and 128 GB DRAM on the end-to-end benchmark using synthetic dataset. Figure 10(c) shows total number of signal events processed per second against the number of parallel threads of data pipeline execution. We observe that LifeStream provides up to $6.02 \times$ better scalability than Trill and $1.90 \times$ better than numerical libraries. This shows that LifeStream can maintain its performance benefits on multi-core parallel data pipeline execution compared to Trill and provide better parallel processing capabilities than numerical library-based approach.

We also observe that Trill goes out of memory and crashes when we run experiments with more than 12 parallel threads. LifeStream, on the other hand, is much more memory-efficient and can scale up to 32 parallel threads as the memory required for the intermediate results are preallocated are reused throughout query execution. Numerical library-based implementation is observed to scale up to 48 threads; however, the performance gets saturated after 24 threads and exhibits a peak performance that is $44 \%$ lower than that of LifeStream.

We also measure the scalability of LifeStream on a multi-machine setup using up to 16 Amazon EC2 m5a.8xlarge [4] machines with each running 12, 24 and 32 parallel threads respectively for Trill, numerical libraries, and LifeStream, since these thread counts are observed to provide the peak performance from multi-core experiment. Figure $10(\mathrm{~d})$ shows the throughput measured against the number of machines. On 16 machines, LifeStream can process 473.66 million events per second which is $8.38 \times$ higher than the peak performance of Trill and $1.73 \times$ higher than that of numerical libraries. This shows that LifeStream can maintain the performance benefits at large scale through efficient data-parallel processing.

\section{RELATED WORK}

Several major solutions were proposed in the past to address large scale stream processing demands [3, 34, 54, 59]. Unfortunately, these solutions fail to be a good fit for physiological data processing due to the unique requirements of (i) programmability or (ii) performance (or sometimes even both). Below, we provide a detailed comparison of LifeStream against key prior works based on these two aspects.

Stream Processing Engines Popular stream processing engines such as Apache Spark stream [5], Storm [54], Flink [10], and Beam [3] provide simple declarative programming interfaces for writing complex data processing pipelines. However, most of them fail to support several features essential for physiological data processing. For instance, Storm [54] does not have any implicit notion of event time or windowing. Spark streaming, on the other hand, does not have support for millisecond precision event time as required for many signals in physiological data and lacks several useful temporal primitive operations that are necessary for writing queries on physiological data. Additionally, as we show in Section 3, these solutions are primarily designed for distributed setup and showcase poor single machine performance.

Trill [11] is the closest streaming engine we could find that provides rich support for temporal operations, high precision event time, and flexible windowing, as well as $1-2$ orders of magnitude higher single machine performance than distributed streaming engines. Unlike Trill, LifeStream takes advantage of the end-to-end locality of the entire data pipeline using locality tracing. Additionally, LifeStream employs optimizations like static memory allocation and targeted query processing to minimize runtime memory 

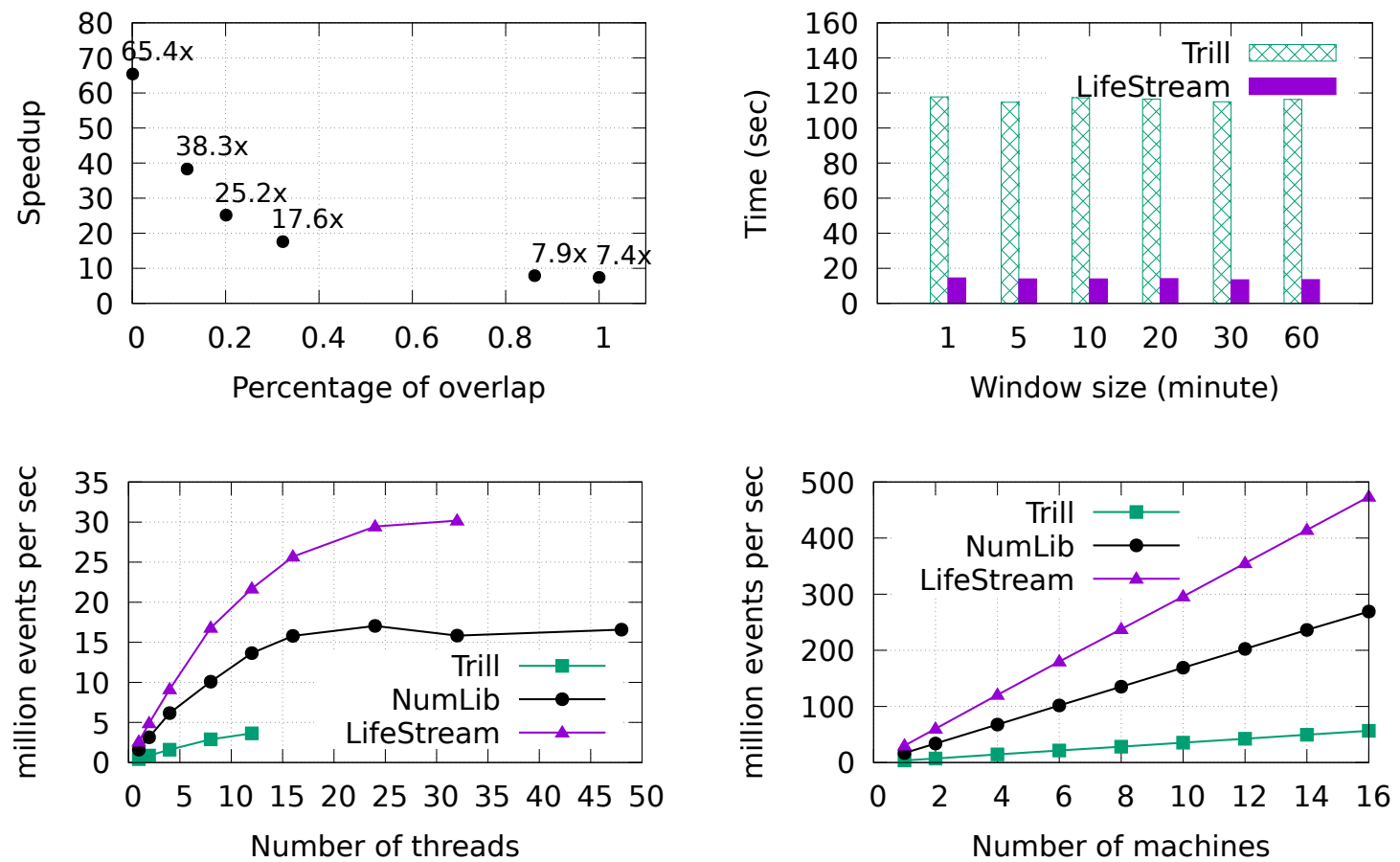

Figure 10: (a) Targeted query processing, (b) Varying window size, (c) Multi-core scaling, (d) Multi-machine scaling

allocation/deallocation overhead and pruning redundant computations. As we have shown in Section 8, these optimizations make LifeStream significantly faster than strong baselines like Trill.

A follow-up work called TrillDSP [35] extends Trill query language interface to support digital signal processing (DSP) operations like Fast Fourier Transform (FFT). Both LifeStream and TrillDSP have similar motivations and they both deal with periodic data streams. However, TrillDSP is primarily focused on providing a programming interface that is suitable for people who are familiar with common signal processing libraries in R and MATLAB, simultaneously supporting stream processing capabilities of Trill without having to make expensive communications with these external libraries. LifeStream proposes a more general solution which addresses both programmability and performance aspects of periodic stream processing. Moreover, the proposed query optimizations in our work, namely locality tracing, static memory allocation, and targeted query processing are unique contributions of LifeStream.

Following the footsteps of Trill, there are two other streaming engines recently proposed, StreamBox [29] and StreamBox-HBM [28] that focus on improving the single machine performance. However, both these designs provide a very low-level and generic programming abstraction and lack a rich high-level temporal language support. Additionally, StreamBox-HBM was designed specifically for machines with high bandwidth memory (HBM), which are both very $\operatorname{rare}^{4}$ and expensive. Compared to these two engines,

${ }^{4}$ In fact, Intel's Knight Landing architecture used in StreamBox-HBM is discontinued now [21].
LifeStream provides a much simpler programming interface with high performance on commodity hardware.

Numerical Libraries There have been some recent studies [39, 41] to improve the performance of numerical library-based data processing pipelines. Weld [39] proposed a compiler-based approach to optimize across disjoint libraries and functions with the help of an intermediate representation (IR). The followup work, called Split Annotations [41], eliminates the need for an IR and re-implementation of library functions while potentially providing similar end-to-end performance benefits.

Even though these solutions can improve the end-to-end performance of numerical library-based data processing pipelines by some margin, the lack of temporal logic support and unified API specification still make such approaches less desirable in terms of ease of programming and maintainability. We believe high-level temporal query language provided by LifeStream is more systematic and appropriate approach for doing data processing on physiological data.

Time-series Databases There are several databases specially designed to store and process time-series datasets efficiently. However, we find time-series databases like InfluxDB [23] and KDB [50] to be a poor fit for physiological data processing. For instance, InfluxDB does not follow relational data model. Therefore, it does not have flexible temporal Join support, a common operation in the physiological data processing. Moreover, these databases only provide limited windowing support. $\mathrm{KDB}$, on the other hand, requires the 
entire dataset to be in memory, which is not feasible as we are dealing with datasets of size up to $1 \mathrm{~TB}$. Additionally, KDB uses a highly esoteric language called $q$ [49] for writing queries in contrast to a more familiar SQL-like language.

We observe that time-series databases are sub-optimal choice from a performance perspective as well. InfluxDB introduces about $2.25 \times$ overhead even on simple large-scale data read operation compared to stream processing engines. We expect even worse performance for more complicated operations.

Stream Programming Languages There has been prior work proposing programming language and compiler infrastructure for writing stream-based computation. Sisal [14] and StreamIt [51] are notable examples of a domain-specific language for writing streaming applications. However, these languages are not designed for temporal data processing and do not implicitly support event time. For example, temporal joining of two independent streams is not natively supported in both StreamIt and Sisal. Moreover, the optimizations in StreamIt are limited to DSP applications whereas the query language and the optimizations proposed in LifeStream apply to generic temporal queries.

\section{CONCLUSION}

In this paper, we showcase the limitations of modern streaming engines and numerical libraries in building complex physiological data processing pipelines regarding their ease of programming, maintainability, and performance. We subsequently propose LifeStream, which provides a simple and flexible temporal query language as the programming interface, and exploits the periodic nature of the physiological data to provide high performance. We propose three key optimizations in LifeStream, namely, (i) locality tracing for improving end-to-end cache utilization of the data pipeline, (ii) memory footprint estimation for minimizing runtime memory allocation and deallocation overhead, and (iii) targeted query processing for pruning redundant computation. We conduct experiments and evaluations on real datasets and use cases, and demonstrate that LifeStream outperforms state-of-the-art streaming engine Trill by as much as $7.5 \times$ and numerical library-based approaches by as much as $3.2 \times$ on the end-to-end data processing performance.

\section{ACKNOWLEDGMENTS}

We first thank our shepherd Abhishek Bhattacharjee and the anonymous reviewers for their valuable feedback and comments. We also like to thank members of the Laussen Lab, especially Mjaye Mazwi, Sebastian Goodfellow, Danny Eytan, Carson McLean, Sana Tonekaboni, Xi Huang, Aslesha Pokhrel, Sujay Nagaraj, and Bobby Greer for providing access to the physiological datasets at The Hospital for Sick Children and helping us build the benchmark suite. We further extend our gratitude to Jinliang Wei and the members of the EcoSystem lab, especially Shang Wang and Geoffrey Yu, for providing insightful comments and constructive feedback on the paper. This project was supported in part by the Canada Foundation for Innovation JELF grant, AWS machine learning research award, NSERC Discovery and CRD grants, and also a grant from Huawei.

\section{A ARTIFACT APPENDIX}

\section{A.1 Abstract}

This artifact includes the source code of LifeStream and instructions to reproduce the main paper's key performance results. We identify the operations and end-to-end benchmark results described in Sections 8.2 and 8.3 as the key results of the paper. The instructions provided are for reproducing the results shown in Figure 9(b) and 9(c) on a synthetically generated data set. We include only the synthetic data set in the artifact since access to the real data set cannot be provided as it is proprietary to The Hospital for Sick Children, Toronto, Canada. The performance results produced on the synthetic data set should be an accurate estimate of the results on the real data set. Moreover, it should be noted that the results from this artifact are going to be better than the ones reported in the main paper as we have improved our results since the original submission.

The artifact can be executed over any runtime environment with .NET Core SDK 3.1 and Python3. However, the instructions provided in this appendix assumes a Ubuntu 20.04 machine. We also provide a docker file to automatically set up the runtime environment for running the artifact. We recommend using the docker environment for running the experiments.

\section{A.2 Artifact Checklist}

- Algorithm: Not applicable

- Program: Custom benchmarks included in the artifact.

- Compilation: C\# compiler with .NET Core 3.1 SDK

- Transformations: No transformation tools required.

- Binary: Source code and scripts included to build and run the binaries.

- Data set: The synthetic data set used in the main paper is provided.

- Run-time environment: .NET Core 3.1 SDK and Python3.

- Hardware: A single general purpose CPU.

- Runtime state: Not sensitive to runtime state.

- Execution: Less than an hour to evaluate all the benchmarks.

- Metrics: Data processing time in seconds.

- Output: Plots similar to Figure 9(b) and 9(c) in the main paper.

- Experiments: Bash script and docker file are provided to run the benchmarks. Numerical variations in the results are negligible.

- How much disk space required (approximately)?: Approximately $250 \mathrm{MB}$.

- How much time is needed to prepare workflow (approximately)?: Approximately 10 minutes to setup the runtime environment.

- How much time is needed to complete experiments (approximately)?: Approximately 30 minutes.

- Publicly available?: Yes

- Code licenses (if publicly available)?: MIT License

- Data licenses (if publicly available)?: Not applicable.

- Workflow framework used?: No.

- Archival link: https://doi.org/10.5281/zenodo.4331660 


\section{A.3 Description}

A.3.1 How to Access. The artifact can be downloaded either from the GitHub link https://github.com/anandj91/LifeStream or from the DOI link https://doi.org/10.5281/zenodo.4331660 .

A.3.2 Hardware Dependencies. LifeStream does not require any special hardware. A single general purpose $\mathrm{CPU}$ would be sufficient for running the artifact.

A.3.3 Software Dependencies. This artifact requires a runtime environment with .NET Core SDK 3.1 and Python3 installed. There are no restrictions on the choice of operating system. However, the instructions provided below assumes Ubuntu 20.04. Additionally, we also include a docker file to automatically setup the runtime environment.

A.3.4 Benchmarks and Baselines. This artifact uses the operations benchmark (Table 3) and end-to-end application benchmark (Figure 3) used in the main paper. We include scripts to reproduce the results on Trill, numerical libraries (NumLib), and LifeStream for these benchmarks. We use execution time as the comparison metric for all the benchmarks.

A.3.5 Data Sets. In the main paper, we use two data sets: a synthetic data set and the real data set collected from The Hospital for Sick Children. The hospital regulations prevent us from sharing the real data set. Therefore, we only include the synthetic data set in the artifact which contains artificially generated $500 \mathrm{~Hz}$ and 125 $\mathrm{Hz}$ signal events. The results produced on the synthetic data set should give an accurate estimate of the results on real data set. For the operations benchmark, we use 1000 minute $500 \mathrm{~Hz}$ data set. End-to-end benchmark is evaluated on data set with both $500 \mathrm{~Hz}$ and $125 \mathrm{~Hz}$ signals with varying data sizes.

\section{A.4 Installation}

This section describes two ways for setting up the runtime environment; docker setup and manual setup. We recommend using docker environment for the experiments. First, download and unpack the artifact and go to the root directory.

For setting up the docker environment,

(1) Build the docker image from the docker file by running the script setup_docker.sh.

For manually setting up the runtime environment,

(1) Go to src directory under the root directory.

(2) Install required packages by running setup. sh script.

\section{A.5 Experiment Workflow}

Once the runtime environment has been properly setup, the following scripts can be used to run the benchmarks on Trill, numerical libraries (NumLib), and LifeStream.

For running in the docker setup,

(1) Run run_docker. sh script.

For running in the manual setup,

(1) Go to src directory under the root directory.

(2) Run run. sh script.

The above scripts run all the benchmarks one after the other and report the execution time for each benchmark on Trill, numerical libraries (NumLib), and LifeStream to the standard output as well as to respective files in the src/results directory.

\section{A.6 Evaluation and Expected Results}

Once the script has finished execution, they would have generated plots similar to Figure 9(b) and 9(c). The figures can be found in the src/results directory under the names op. pdf and e2e.pdf.

The plotted figures should show the performance comparison of LifeStream against Trill and numerical libraries (NumLib) on the synthetic data set. The results reported in the main paper, on the other hand, is based on the real data set, which we cannot share due to privacy concerns. Hence the evaluators should be comparing the relative performance of LifeStream against Trill and NumLib rather than the absolute numbers.

Compared to Trill, LifeStream is expected to perform $7-8 \times$ better on end-to-end benchmark and $5-11 \times$ better on operations benchmarks. Compared to NumLib, LifeStream should showcase performance improvement up to $\sim 3 \times$ on normalize, resample, and passfilter benchmarks in operations benchmark. Results of LifeStream on fillconst and fillmean are expected to be worse than NumLib by up to $\sim 2 \times$ as reported in the main paper. It should be noted that the performance results of LifeStream on resample and passfilter benchmarks have been improved since the original submission as a result of several additional optimizations we incorporated. Finally, LifeStream should perform $2-3 \times$ better than NumLib on the end-to-end benchmark.

\section{A.7 Experiment Customization}

To run a specific benchmark under manual setup, run_bench.sh script under the src directory can be executed by passing the data set size (in seconds), execution engine, and the benchmark as follows.

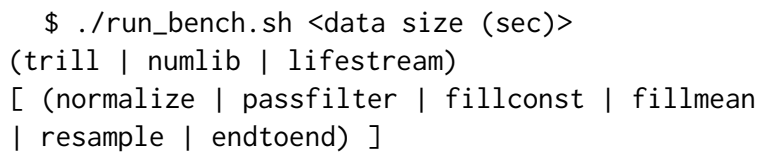

run_bench.sh script should report the execution time for the specific benchmark to the standard output.

To run a specific benchmark under the docker setup, open a shell to the docker container from the root directory using the following command before running the run_bench. sh script.

\footnotetext{
\$ docker run -it - $v$ \$PWD/src/results:/root/results lifestream_image/bin/bash
}

\section{A.8 Methodology}

This artifact follows the following submission, reviewing and badging methodology:

- www.acm.org/publications/policies/artifact-review-badging

- http://cTuning.org/ae/submission-20201122.html

- http://cTuning.org/ae/reviewing-20201122.html 


\section{REFERENCES}

[1] Karim Abouelmehdi, Abderrahim Beni-Hessane, and Hayat Khaloufi. 2018. Big healthcare data: preserving security and privacy. Fournal of Big Data 5, 1 (09 Jan 2018), 1. https://doi.org/10.1186/s40537-017-0110-7

[2] George Adam, Ladislav Rampášek, Zhaleh Safikhani, Petr Smirnov, Benjamin Haibe-Kains, and Anna Goldenberg. 2020. Machine learning approaches to drug response prediction: challenges and recent progress. npj Precision Oncology 4, 1 (15 Jun 2020), 19. https://doi.org/10.1038/s41698-020-0122-1

[3] Tyler Akidau, Robert Bradshaw, Craig Chambers, Slava Chernyak, Rafael J. Fernández-Moctezuma, Reuven Lax, Sam McVeety, Daniel Mills, Frances Perry, Eric Schmidt, and Sam Whittle. 2015. The Dataflow Model: A Practical Approach to Balancing Correctness, Latency, and Cost in Massive-Scale, Unbounded, Out-ofOrder Data Processing. Proceedings of the VLDB Endowment 8 (2015), 1792-1803.

[4] Amazon. [n.d.]. EC2 m5a.8xlarge. https://aws.amazon.com/ec2/instance-types/ $\mathrm{m} 5 /$

[5] Michael Armbrust, Tathagata Das, Joseph Torres, Burak Yavuz, Shixiong Zhu, Reynold Xin, Ali Ghodsi, Ion Stoica, and Matei Zaharia. 2018. Structured Streaming: A Declarative API for Real-Time Applications in Apache Spark. In Proceedings of the 2018 International Conference on Management of Data (Houston, TX, USA) (SIGMOD '18). Association for Computing Machinery, New York, NY, USA, 601-613. https://doi.org/10.1145/3183713.3190664

[6] Brian Babcock, Shivnath Babu, Mayur Datar, Rajeev Motwani, and Jennifer Widom. 2002. Models and Issues in Data Stream Systems. In Proceedings of the Twenty-First ACM SIGMOD-SIGACT-SIGART Symposium on Principles of Database Systems (Madison, Wisconsin) (PODS '02). Association for Computing Machinery, New York, NY, USA, 1-16. https://doi.org/10.1145/543613.543615

[7] Valentina Baljak, Adis Ljubovic, Jonathan Michel, Mason Montgomery, and Richard Salaway. 2018. A scalable realtime analytics pipeline and storage architecture for physiological monitoring big data. Smart Health 9 (2018), 275-286.

[8] Jan N. Basile. 2002. Systolic blood pressure. BMF (Clinical research ed.) 325, 7370 (26 Oct 2002), 917-918. https://doi.org/10.1136/bmj.325.7370.917 12399325 [pmid].

[9] beckershospitalreview.com. [n.d.]. 28 health system cyberattacks, data breaches so far in 2020. https://www.beckershospitalreview.com/cybersecurity/28-healthsystem-cyberattacks-data-breaches-so-far-in-2020.html

[10] Paris Carbone, Asterios Katsifodimos, Stephan Ewen, Volker Markl, Seif Haridi, and Kostas Tzoumas. 2015. Apache Flink ${ }^{\mathrm{TM}}$ : Stream and Batch Processing in a Single Engine. IEEE Data Eng. Bull. 38 (2015), 28-38.

[11] Badrish Chandramouli, Jonathan Goldstein, Mike Barnett, Robert DeLine, Danyel Fisher, John C. Platt, James F. Terwilliger, and John Wernsing. 2014. Trill: A High-Performance Incremental Query Processor for Diverse Analytics. Proc. VLDB Endow. 8, 4 (Dec. 2014), 401-412. https://doi.org/10.14778/2735496.2735503

[12] Calvin J. Chiew, Nan Liu, Takashi Tagami, Ting Hway Wong, Zhi Xiong Koh, and Marcus E. H. Ong. 2019. Heart rate variability based machine learning models for risk prediction of suspected sepsis patients in the emergency department. Medicine 98, 6 (Feb 2019), e14197-e14197. https://doi.org/10.1097/MD. 000000000001419730732136 [pmid].

[13] Ambika Choudhury and Deepak Gupta. 2019. A Survey on Medical Diagnosis of Diabetes Using Machine Learning Techniques: IC3 2018. 67-78. https://doi.org/10. 1007/978-981-13-1280-9_6

[14] John T. Feo, David C. Cann, and Rodney R. Oldehoeft. 1990. A report on the sisal language project. F. Parallel and Distrib. Comput. 10, 4 (1990), 349 - 366 https://doi.org/10.1016/0743-7315(90)90035-N Data-flow Processing

[15] C. M. Furse, R. Harrison, and F. Solzbacher. 2007. Recent Advances in BioMedical Telemetry. In 2007 International Conference on Electromagnetics in Advanced Applications. 1026-1027.

[16] Andrew J Goodwin, Danny Eytan, Robert W Greer, Mjaye Mazwi, Anirudh Thommandram, Sebastian D Goodfellow, Azadeh Assadi, Anusha Jegatheeswaran, and Peter C Laussen. 2020. A practical approach to storage and retrieval of highfrequency physiological signals. Physiological Measurement 41, 3 (apr 2020), 035008. https://doi.org/10.1088/1361-6579/ab7cb5

[17] Kyeonghye Guk, Gaon Han, Jaewoo Lim, Keunwon Jeong, Taejoon Kang, EunKyung Lim, and Juyeon Jung. 2019. Evolution of Wearable Devices with RealTime Disease Monitoring for Personalized Healthcare. Nanomaterials (Basel, Switzerland) 9, 6 (29 May 2019), 813. https://doi.org/10.3390/nano9060813 31146479 [pmid].

[18] Chuanxiong Guo. 2015. Pingmesh: A Large-Scale System for Data Center Network Latency Measurement and Analysis. In SIGCOMM (sigcomm ed.) ACM. https://www.microsoft.com/en-us/research/publication/pingmesh-largescale-system-data-center-network-latency-measurement-analysis/

[19] HHS. [n.d.]. Your Rights Under HIPAA. https://www.hhs.gov/hipaa/forindividuals/guidance-materials-for-consumers/index.html

[20] Melanie Hilario, Alexandros Kalousis, Markus Müller, and Christian Pellegrini 2003. Machine learning approaches to lung cancer prediction from mass spectra PROTEOMICS 3, 9 (2003), 1716-1719. https://doi.org/10.1002/pmic.200300523 arXiv:https://onlinelibrary.wiley.com/doi/pdf/10.1002/pmic.200300523
[21] HPCWire. [n.d.]. Requiem for a Phi: Knights Landing Discontinued. https: //www.hpcwire.com/2018/07/25/end-of-the-road-for-knights-landing-phi/

[22] A. F. Hussein, N. A. kumar, M. Burbano-Fernandez, G. Ramírez-González, E. Abdulhay, and V. H. C. De Albuquerque. 2018. An Automated Remote CloudBased Heart Rate Variability Monitoring System. IEEE Access 6 (2018), 7705577064 .

[23] InfluxData. [n.d.]. InfluxDB time-series database. https://www.influxdata.com/

24] Intel. [n.d.]. Intel vTune profiler. https://software.intel.com/content/www/us/en/ develop/tools/vtune-profiler.html

[25] Alistair E.W. Johnson, Tom J. Pollard, Lu Shen, Li wei H. Lehman, Mengling Feng, Mohammad Ghassemi, Benjamin Moody, Peter Szolovits, Leo Anthony Celi, and Roger G. Mark. 2016. MIMIC-III, a freely accessible critical care database. Scientific Data 3, 1 (24 May 2016), 160035. https://doi.org/10.1038/sdata.2016.35

[26] Jared L. Katzman, Uri Shaham, Alexander Cloninger, Jonathan Bates, Tingting Jiang, and Yuval Kluger. 2018. DeepSurv: personalized treatment recommender system using a Cox proportional hazards deep neural network. BMC Medical Research Methodology 18, 1 (26 Feb 2018), 24. https://doi.org/10.1186/s12874018-0482-1

[27] Frank McSherry, Michael Isard, and Derek G. Murray. 2015. Scalability! But at what COST?. In 15th Workshop on Hot Topics in Operating Systems (HotOS XV). USENIX Association, Kartause Ittingen, Switzerland. https://www.usenix.org/ conference/hotos15/workshop-program/presentation/mcsherry

[28] Hongyu Miao, Myeongjae Jeon, Gennady Pekhimenko, Kathryn S. McKinley, and Felix Xiaozhu Lin. 2019. StreamBox-HBM: Stream Analytics on High Bandwidth Hybrid Memory. In Proceedings of the Twenty-Fourth International Conference on Architectural Support for Programming Languages and Operating Systems (Providence, RI, USA) (ASPLOS '19). Association for Computing Machinery, New York, NY, USA, 167-181. https://doi.org/10.1145/3297858.3304031

[29] Hongyu Miao, Heejin Park, Myeongjae Jeon, Gennady Pekhimenko, Kathryn S. McKinley, and Felix Xiaozhu Lin. 2017. StreamBox: Modern Stream Processing on a Multicore Machine. In 2017 USENIX Annual Technical Conference (USENIX ATC 17). USENIX Association, Santa Clara, CA, 617-629. https://www.usenix. $\mathrm{org} /$ conference/atc17/technical-sessions/presentation/miao

[30] Microsoft. [n.d.]. .NET Core. https://dotnet.microsoft.com/

[31] Microsoft. [n.d.]. .NET Libraries. https://en.wikipedia.org/wiki/List_of numerical_libraries\#.NET_Framework_languages_C.23.2C_F.23.2C_VB.NET_ and_PowerShell

[32] R. Miotto, F. Wang, S. Wang, X. Jiang, and J. T. Dudley. 2018. Deep learning for healthcare: review, opportunities and challenges. Brief. Bioinformatics 19, 6 (11 2018), 1236-1246.

[33] Marion Morel, Catherine Achard, Richard Kulpa, and Sverine Dubuisson. 2018. Time-Series Averaging Using Constrained Dynamic Time Warping with Tolerance. Pattern Recogn. 74, C (Feb. 2018), 77-89. https://doi.org/10.1016/j.patcog. 2017.08.015

[34] Derek G. Murray, Frank McSherry, Rebecca Isaacs, Michael Isard, Paul Barham, and Martín Abadi. 2013. Naiad: A Timely Dataflow System. In Proceedings of the Twenty-Fourth ACM Symposium on Operating Systems Principles (Farminton, Pennsylvania) (SOSP '13). Association for Computing Machinery, New York, NY, USA, 439-455. https://doi.org/10.1145/2517349.2522738

[35] Milos Nikolic, Badrish Chandramouli, and Jonathan Goldstein. 2017. Enabling Signal Processing over Data Streams. In SIGMOD 2017, May 14-19, 2017, Chicago, Illinois, USA (sigmod 2017, may 14-19, 2017, chicago, illinois, usa ed.). ACM. https://www.microsoft.com/en-us/research/publication/enabling-signalprocessing-data-streams/

[36] Silvia Nittel. 2015. Real-Time Sensor Data Streams. SIGSPATIAL Special 7, 2 (Sept. 2015), 22-28. https://doi.org/10.1145/2826686.2826691

[37] Travis Oliphant. [n.d.]. NumPy. https://numpy.org/

[38] Travis Oliphant, Pearu Peterson, and Eric Jones. [n.d.]. SciPy. https://www.scipy. org/

[39] Shoumik Palkar, James Thomas, Deepak Narayanan, Pratiksha Thaker, Rahul Palamuttam, Parimajan Negi, Anil Shanbhag, Malte Schwarzkopf, Holger Pirk, Saman Amarasinghe, Samuel Madden, and Matei Zaharia. 2018. Evaluating End-to-End Optimization for Data Analytics Applications in Weld. Proc. VLDB Endow. 11, 9 (May 2018), 1002-1015. https://doi.org/10.14778/3213880.3213890

[40] Shoumik Palkar, J. Thomas, A. Shanbhag, D. Narayanan, H. Pirk, M. Schwarzkopf, Saman P. Amarasinghe, M. Zaharia, and Stanford InfoLab. 2016. Weld : A Common Runtime for High Performance Data Analytics.

[41] Shoumik Palkar and Matei Zaharia. 2019. Optimizing Data-Intensive Computations in Existing Libraries with Split Annotations. In Proceedings of the 27th ACM Symposium on Operating Systems Principles (Huntsville, Ontario, Canada) (SOSP '19). Association for Computing Machinery, New York, NY, USA, 291-305. https://doi.org/10.1145/3341301.3359652

[42] Fabian Pedregosa, Gaël Varoquaux, Alexandre Gramfort, Vincent Michel, Bertrand Thirion, Olivier Grisel, Mathieu Blondel, Peter Prettenhofer, Ron Weiss, Vincent Dubourg, Jake Vanderplas, Alexandre Passos, David Cournapeau, Matthieu Brucher, Matthieu Perrot, and Édouard Duchesnay. 2011. Scikit-learn: Machine Learning in Python. Journal of Machine Learning Research 12, 85 (2011), 
2825-2830. http://jmlr.org/papers/v12/pedregosa11a.html

[43] Gennady Pekhimenko, Chuanxiong Guo, Myeongjae Jeon, Peng Huang, and Lidong Zhou. 2018. TerseCades: Efficient Data Compression in Stream Processing. In 2018 USENIX Annual Technical Conference (USENIX ATC 18). USENIX Association, Boston, MA, 307-320. https://www.usenix.org/conference/atc18/ presentation/pekhimenko

[44] Phillips. [n.d.]. Phillips Patient Monitoring. https://www.usa.philips.com/ healthcare/solutions/patient-monitoring

[45] readitquik.com. [n.d.]. Top 7 AWS Outages That Wreaked Havoc. https://www. readitquik.com/articles/cloud-3/top-7-aws-outages-that-wreaked-havoc/

[46] Donald Reay. 2015. Finite Impulse Response Filters. 97-162. https://doi.org/10. 1002/9781119078227.ch3

[47] Jonathan G. Richens., Ciarán M. Lee, and Saurabh Johri. 2020. Improving the accuracy of medical diagnosis with causal machine learning. Nature Communications 11, 1 (11 Aug 2020), 3923. https://doi.org/10.1038/s41467-020-17419-7

[48] D. Sculley, Gary Holt, Daniel Golovin, Eugene Davydov, Todd Phillips, Dietmar Ebner, Vinay Chaudhary, Michael Young, Jean-Francois Crespo, and Dan Dennison. 2015. Hidden Technical Debt in Machine Learning Systems. In Proceedings of the 28th International Conference on Neural Information Processing Systems - Volume 2 (Montreal, Canada) (NIPS'15). MIT Press, Cambridge, MA, USA, 2503-2511.

[49] Kx Systems. [n.d.]. Kdb+ and q documentation. https://code.kx.com/q/ref/

[50] Kx Systems. [n.d.]. kdb+ time-series database. https://code.kx.com/q/

[51] William Thies, Michal Karczmarek, and Saman P. Amarasinghe. 2002. StreamIt A Language for Streaming Applications. In Proceedings of the 11th International Conference on Compiler Construction (CC'02), R. Nigel Horspool (Ed.). SpringerVerlag, London, UK, UK, 179-196. https://doi.org/10.1007/3-540-45937-5_14

[52] Sana Tonekaboni, Mjaye Mazwi, Peter Laussen, Danny Eytan, Robert Greer Sebastian Goodfellow, Andrew Goodwin, Michael Brudno, and Anna Goldenberg. 2018. Prediction of Cardiac Arrest from Physiological Signals in the Pediatric ICU.
[53] tripwire.com. [n.d.]. 6 Cloud Security Threats Healthcare Companies May Face With Solutions. https://www.tripwire.com/state- of-security/featured/6-cloudsecurity-threats-healthcare-companies-face-solutions/

[54] Jan Sipke van der Veen, Bram van der Waaij, Elena Lazovik, Wilco Wijbrandi, and Robert J. Meijer. 2015. Dynamically Scaling Apache Storm for the Analysis of Streaming Data. In Proceedings of the 2015 IEEE First International Conference on Big Data Computing Service and Applications (BIGDATASERVICE '15). IEEE Computer Society, USA, 154-161. https://doi.org/10.1109/BigDataService.2015.56

[55] Jenna Wiens and Erica S Shenoy. 2017. Machine Learning for Healthcare: On the Verge of a Major Shift in Healthcare Epidemiology. Clinical Infectious Diseases 66, 1 (08 2017), 149-153. https://doi.org/10.1093/cid/cix731 arXiv:https://academic.oup.com/cid/article-pdf/66/1/149/24265881/cix731.pdf

[56] Wikipedia. [n.d.]. Dynamic Time Warping. https://en.wikipedia.org/wiki/ Dynamic_time_warping

[57] Wikipedia. [n.d.]. Imputation (statistics). https://en.wikipedia.org/wiki/ Imputation_(statistics)

[58] Wikipedia. [n.d.]. Linear interpolation. https://en.wikipedia.org/wiki/Linear interpolation

[59] Matei Zaharia, Reynold S. Xin, Patrick Wendell, Tathagata Das, Michael Armbrust, Ankur Dave, Xiangrui Meng, Josh Rosen, Shivaram Venkataraman, Michael J. Franklin, Ali Ghodsi, Joseph Gonzalez, Scott Shenker, and Ion Stoica. 2016. Apache Spark: A Unified Engine for Big Data Processing. Commun. ACM 59, 11 (Oct. 2016), 56-65. https://doi.org/10.1145/2934664

[60] Wenting Zheng, Ankur Dave, Jethro G. Beekman, Raluca Ada Popa, Joseph E. Gonzalez, and Ion Stoica. 2017. Opaque: An Oblivious and Encrypted Distributed Analytics Platform. In 14th USENIX Symposium on Networked Systems Design and Implementation (NSDI 17). USENIX Association, Boston, MA, 283-298. https: //www.usenix.org/conference/nsdi17/technical-sessions/presentation/zheng 